\title{
Improving excited state potential energy surfaces via optimal orbital shapes
}

\author{
Lan Nguyen $\operatorname{Tran}^{1,2, *}$ and Eric Neuscamman ${ }^{1,3, \dagger}$ \\ ${ }^{1}$ Department of Chemistry, University of California, Berkeley, California, 94720, USA \\ ${ }^{2}$ Ho Chi Minh City Institute of Physics, VAST, Ho Chi Minh City 700000, Vietnam \\ ${ }^{3}$ Chemical Sciences Division, Lawrence Berkeley National Laboratory, Berkeley, CA, 94720, USA
}

(Dated: May 24, 2022)

\begin{abstract}
We demonstrate that, rather than resorting to high-cost dynamic correlation methods, qualitative failures in excited state potential energy surface predictions can often be remedied at no additional cost by ensuring that optimal molecular orbitals are used for each individual excited state. This approach also avoids the weighting choices required by state-averaging and dynamic weighting and obviates their need for expensive wave function response calculations when relaxing excited state geometries. Although multi-state approaches are of course preferred near conical intersections, other features of excited state potential energy surfaces can benefit significantly from our singlestate approach. In three different systems, including a double bond dissociation, a biologically relevant amino hydrogen dissociation, and an amino-to-ring intramolecular charge transfer, we show that state-specific orbitals offer qualitative improvements over the state-averaged status quo.
\end{abstract}

Introduction. Exited-state geometry relaxations are an essential phenomenon in molecular photochemistry. Alongside inter-state properties like non-adiabatic couplings and transition dipoles, single-state potential energy surface (PES) features like the depths and locations of excited-state minima and the heights of barriers between minima help determine how a molecule will respond and transform under exposure to light. For example, selecting ligands in order to extend the lifetimes of Fe-based metal-to-ligand charge-transfer (MLCT) states depends centrally on the relative energies and positions of the MLCT and other excited state minima. ${ }^{1-5}$ While density functional theory (DFT), with its low cost and often excellent ground state energetics, is widely used in studying excited states, its well-known difficulties with charge-transfer states and in systems exhibiting strong electron correlations make reliable, low-cost alternatives a high priority in the study of photochemistry. The complete active space self-consistent field (CASSCF) approach is often a powerful alternative,$^{6-8}$ but it is significantly more difficult to use than DFT, and, due to its own limitations, can still make qualitative errors in PESs without the aid of expensive post-CASSCF correlation corrections. A good example of this frustrating reality occurs in the charge transfer state of 4-aminobenzonitrile $(\mathrm{ABN})$, where state-averaging compromises prevent the wave function from undergoing proper orbital relaxation and lead CASSCF to predict a qualitatively incorrect excited state geometry. ${ }^{9}$ Although applying corrections with complete active space perturbation theory (CASPT2) brings predictions in line with experiment, ${ }^{9}$ such post-CASSCF correlation methods greatly increase computational cost. While such methods' incorporation of the finer details of electron correlation is essential for high-precision energetics, we show here that in this case and others, qualitatively correct predictions can be restored simply by making the molecular orbital shapes optimal for individual excited states.

While the widely used practice of state averaging (SA) has seen many successes in excited state investigations, it faces a number of important challenges. In the SA approach, the molecular orbital shapes are chosen by minimizing the weighted average of multiple states' energies. This appears at first glace to be balanced, and in many cases is, but can also entrench imbalances if the needs of one state (e.g. strong orbital relaxations following charge transfer) are denied in favor of the needs of others (e.g. multiple local excitations that should not involve strong orbital relaxations). As we discuss below, this problem appears to be responsible for SA-CASSCF's failure to predict an excitation-induced twist in ABN. SA-CASSCF is also a popular way to address the challenge of root flipping, where an optimization fails to converge due to two states exchanging back and forth in the energy ordering. However, SA is not a panacea here, as there is always the risk of the highest-energy state in the average flipping with the next state not included in the average. Glover, for example, has recently shown explicitly that SA-CASSCF still suffers from root flipping. ${ }^{10}$ Another challenge is that SA-CASSCF often introduces discontinuities in potential energy surfaces (PESs), ${ }^{10-13}$ which can be a particular problem for dynamics simulations. ${ }^{10,14}$ Whether these discontinuities arise from the highest state in the average crossing the lowest state not in the average as the atoms move around, or from a cusp in a single state being converted to a discontinuity in all states via the SA link, this high-priority problem has attracted a good deal of attention. One approach to address the issue is to prepare good orbital sets for complete active space configuration interaction (CASCI), such as floating occupation molecular orbitals (FOMO), ${ }^{15}$ and then forgoing the SA-CASSCF orbital optimization entirely. While less prone to PES discontinuities, this approach unfortunately can still produce them for high-lying excited states. ${ }^{16}$ An alternative approach, and one that is particularly relevant near conical intersections where retaining at least a two-state treatment is advantageous, is to weight the SA dynamically so as to favor the needs of the important states while retaining the stability offered by SA. ${ }^{10-12}$ The draw- 
back is that the now energy-dependent weights complicate the evaluation of analytic gradients even more than SA does already. ${ }^{10,12}$ Ideally, discontinuities and root flipping would be avoided while retaining ground-state CASSCF's simple analytic gradients, each state would have the orbital relaxations it needs, and the user would not need to make any decisions about weightings that affect the final predictions.

Towards these ends, we present in this paper a study of how fully excited-state-specific CASSCF, in which the molecular orbitals are optimized solely for the benefit of the state in question and collapse to lower states is avoided through careful state-tracking methods, ${ }^{13}$ can achieve these goals and succeed in cases where SACASSCF suffers qualitative failures. In addition to the case of $\mathrm{ABN}$, where experiment and expensive postCASSCF methods predict a twisted charge transfer geometry while SA-CASSCF does not, ${ }^{17,18}$ we investigate the crossing of analine's first ${ }^{1} \pi \pi^{*}$ and charge transfer states (which SA-CASSCF fails to predict ${ }^{19,20}$ ) and PES disappearance and discontinuity in thioacetone. In addition to qualitative improvements in accuracy, it is important to emphasize that the excited-state-specific approach, by making the state's energy stationary with respect to the orbital shapes, avoids the response calculations that SA-CASSCF and dynamic-weighting need to perform ${ }^{21}$ when evaluating analytic gradients. Avoiding the response evaluations matters, as they are more expensive $^{22}$ and can be more prone to convergence issues $^{23}$ than CASSCF itself. In our approach, analytic gradients are no more difficult than in the ground state case, and indeed can use the exact same gradient code. While these advantages in both accuracy and numerical simplicity are exciting, we should keep in mind that SA-CASSCF is popular precisely because it is often successful, and so it is important to emphasize the particular situations in which it is most in need of assistance. In this regard, we will point to charge transfer, which is a technologically important case where SA is at particular risk of entrenching biases between states rather than creating balance. ${ }^{13,24,25}$

Theory. Our state-specific approach (SS-CASSCF) rests on the general property that exact Hamiltonian eigenstates are energy stationary points. In the context of an approximate ansatz like CASSCF, the idea is to find the ansatz's stationary point that corresponds to the excited state under study. For simplicity, we retain the two-step approach common to many CASSCF implementations in which the orbitals and CI variables are optimized separately. In particular, the orbitals are optimized so as to minimize the energy gradient norm ${ }^{13}$ (rather than the energy, which would encourage collapse towards the ground state), while the CI variables are chosen at each stage as the CASCI root most similar to the desired state. While there are of course many ways to make a precise definition for what one means by similar, we have found ${ }^{13}$ that the measure

$$
Q_{W \Gamma}=\frac{\left\langle\Psi\left|(\omega-\hat{H})^{2}\right| \Psi\right\rangle}{\langle\Psi \mid \Psi\rangle}+\frac{\left\|\Gamma_{t}-\Gamma\right\|}{n_{\mathrm{CAS}}}
$$

is particularly effective. Here $\omega$ is a target energy that we think is close to the energy of the state we are after, $\Gamma$ is our state's one-body density matrix, $\Gamma_{t}$ is a target density matrix (taken either from an initial CASCI or from the previous iteration), and $n_{C A S}$ is the number of active orbitals. The idea is that, after finding the lowenergy roots of the CASCI problem, we select the root with the lowest value for $Q_{W \Gamma}$ and then perform an orbital optimization step that seeks to make that root's energy stationary with respect to orbital changes. Crucially, the selection of the root based on its energy and density matrix makes the approach insensitive to root flipping, so even in cases where the order of the states in the CASCI changes as the orbitals are optimized, the approach converges to the energy stationary point corresponding to the desired excited state. ${ }^{13}$ Note especially that the precise choices for $\omega$ and $\Gamma_{t}$ do not affect the final outcome, so long as they lead the optimization to converge to the correct CASSCF stationary point. In other words, the final energy depends only on the wave function at the energy stationary point, which is independent of $\omega$ and $\Gamma_{t}$, but made easier to find and converge to via intelligent choices for these parameters.

Once the energy is stationary, the Hellman-Feynman theorem guarantees that analytic gradients with respect to nuclear coordinates $R$ simplify to

$$
\frac{d E}{d R}=\left\langle\Psi\left|\frac{\partial \hat{H}}{\partial R}\right| \Psi\right\rangle
$$

in a direct parallel to the situation for ground states. There is no need for response calculations, as full energy stationarity eliminates the wave function response terms from the gradient expression. In SA-CASSCF, in contrast, the energies of individual states that control the PES are not stationary (it is only their weighted average that is) and so additional terms involving the wave function's response to the geometry distortion appear in the gradient equation and must be evaluated. Our analytic gradients in hand, we perform our geometry relaxations using the geomopt module within pySCF, ${ }^{26}$ which interfaces with geomeTRIC ${ }^{27}$ and PyBerny ${ }^{28}$ for constrained and unconstrained geometry optimizations, respectively. Comparison calculations with SA-CASSCF were performed using Molpro. ${ }^{29}$

Results. Let us begin with the relatively simple example of $\mathrm{C}-\mathrm{S}$ bond photodissociation, which can be important in astrochemistry ${ }^{30}$ biomedicine, ${ }^{31}$ and catalysis. ${ }^{32}$ For C-S single bond dissociation, non-radiative internal conversion between the first excited state ${ }^{1} \pi \pi^{*}$ and the dissociative ${ }^{1} \pi \sigma^{*}$ state is key. ${ }^{33}$ Here we instead investigate the $\mathrm{C}-\mathrm{S}$ double bond dissociation of thioacetone, $\left(\mathrm{CH}_{3}\right)_{2} \mathrm{CS}$, using both SA-CASSCF and SS-CASSCF. By performing geometry relaxations for each of the low-lying 


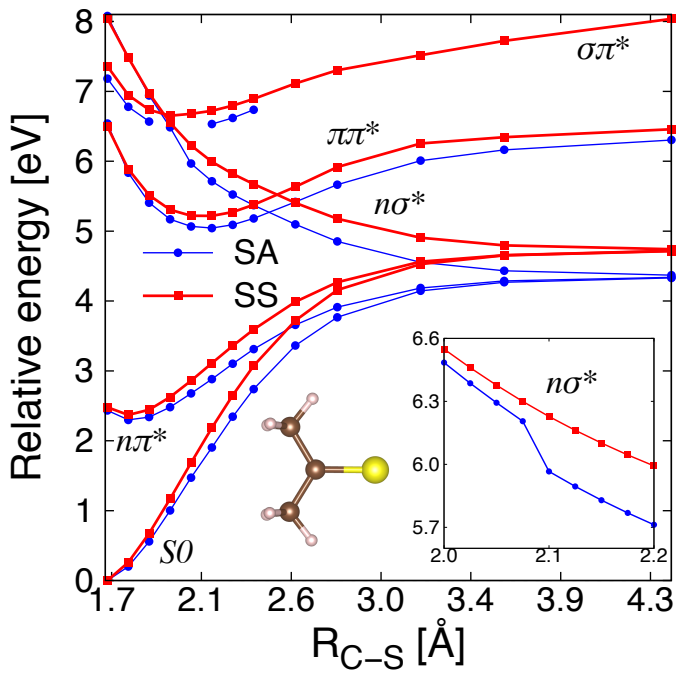

FIG. 1. Potential energy curves showing, for each low-lying singlet state of thioacetone, the energy of the state after relaxing the geometry with the C-S bond distance $R_{C-S}$ held fixed. SA-CASSCF used a 5-state SA with equal weights, while both SA-CASSCF and SS-CASSCF employed the 6$31 \mathrm{G}(\mathrm{d})$ basis and a $(6 \mathrm{e}, 5 \mathrm{o})$ active space containing the $\sigma, \sigma^{*}$, $\pi, \pi^{*}$, and lone pair $n$ orbitals.

singlet states at a series of fixed $\mathrm{C}-\mathrm{S}$ bond distances, we can see which states are expected to be key for the double bond dissociation. We see in Figure 1 that, among the low-lying singlets, it is the ${ }^{1} n \sigma^{*}$ state that is dissociative in character, while the ${ }^{1} n \pi^{*},{ }^{1} \pi \pi^{*}$, and ${ }^{1} \sigma \pi^{*}$ surfaces are all bound. For the lowest four states, the SA and SS approaches are in qualitative agreement, but SS-CASSCF displays a clear advantage for the ${ }^{1} \sigma \pi^{*}$ state. For this state, the SA-CASSCF geometry optimization was unable to converge at most $\mathrm{C}-\mathrm{S}$ bond distances, while SSCASSCF optimizations converged at all distances. While it is possible that extending the SA to include more than five states could help here, this is highly undesirable, as including more states leads to each state having even less say in how the orbitals should be shaped, and, worse, increases the chances of finding discontinuities, as a discontinuity in any states' surface gets spread to all states through their link in the SA energy. With five states in the average, SA-CASSCF suffers one discontinuity already, which the inset of Figure 1 shows near the $R_{C-S}=2.1 \AA$ geometry of the ${ }^{1} n \sigma^{*}$ state. (Note that, although at that geometry all states show a discontinuity due to the SA link, this is not seen in the figure, as the other states' energies are being reported at their own relaxed geometries). The discontinuity is relatively small, and does not alter the basic dissociative character of the state, but even small discontinuities can strongly affect dynamics simulations. ${ }^{10,12}$ In the SS approach, in contrast, the ${ }^{1} \sigma \pi^{*}$ and ${ }^{1} n \sigma^{*}$ states are quite well behaved, and we need not agonize over how many states to include in any average and how this may affect orbital quality.
With the molecular orbital shapes now optimal for each state individually, these difficulties are avoided, the geometry optimizations all converge successfully, and no discontinuities are encountered.

We now turn our attention to aniline, $\left(\mathrm{C}_{6} \mathrm{H}_{5} \mathrm{NH}_{2}\right)$, a common basis unit for biomolecules whose $\mathrm{N}-\mathrm{H}$ photodissociation is important for medical applications, including UV radiation protection within sunscreen. ${ }^{34}$ Aniline's excited-state dynamics have been extensively studied, ${ }^{19,20,34-38}$ and, as is common for heteroaromatic biomolecules, the ${ }^{1} \pi \sigma^{*}$ state has been shown to facilitate the photodissociation process. ${ }^{34}$ It is particularly noteworthy that this state is quasi-bound in the region of the S0 minimum, with dissociative character only appearing once the $\mathrm{N}-\mathrm{H}$ bond has been stretched. Equation of motion coupled cluster (EOM-CCSD) predicts the barrier to leave the quasi-bound region and dissociate to be $0.5 \mathrm{eV},{ }^{37}$ and experimental investigations suggest that the first ${ }^{1} \pi \pi^{*}$ excited state intersects the ${ }^{1} \pi \sigma^{*}$ state near the latter's quasi-bound local minimum geometry. ${ }^{35}$ Previous theoretical investigation has failed to predict this crossing using SA-CASSCF, ${ }^{19,20}$ and it is only when expensive post-CASSCF methods like extended multiconfiguration quasi-degenerate second-order perturbation theory ${ }^{19}$ (XMCQDPT2) and extended multi-state multi-reference perturbation theory ${ }^{20}$ (XMS-CASPT2) are employed that a crossing is predicted. With the ${ }^{1} \pi \sigma^{*}$ state showing a substantial change in dipole moment compared to the ground state and thus possessing at least some charge transfer character, we wondered whether predicting the crossing really required such expensive methodology or, instead, whether this was a case in which states with substantially different needs in terms of molecular orbital shapes were being ill-served by the compromises inherent to state averaging.

As seen in Figure 2, our SA-CASSCF calculations also fail to predict a crossing, whereas SS-CASSCF predicts a crossing near an $\mathrm{N}-\mathrm{H}$ bond distance of $0.95 \AA$. Although the crossing is predicted by post-CASSCF methods to occur closer to a bond distance of $1.05 \AA$, showing that post-CASSCF correlation effects do play a quantitative role here, the fact that SS-CASSCF predicts a crossing at all is a qualitative improvement over the SA approach. We also note that, although it is a smaller advantage, the SS-CASSCF dissociation barrier of $0.49 \mathrm{eV}$ is closer to the $0.5 \mathrm{eV}$ EOM-CCSD prediction ${ }^{37}$ than is the $0.58 \mathrm{eV}$ barrier of SA-CASSCF. To understand why SA-CASSCF has difficulty here, we have plotted the $\sigma^{*}$ orbital from our SS-CASSCF ${ }^{1} \pi \sigma^{*}$ state in Figure 3 , where we see that the charge transfer character suggested by the large dipole change is really a Rydberg-like extension of the $\sigma^{*}$ orbital off one side of the molecule. The large change in dipole is nonetheless present, which creates a statespecific need for the non-active orbitals to respond and re-polarize their electron distributions. This effect is difficult to achieve in SA-CASSCF, as these re-polarizations are inappropriate for the other two states. We find that, even if we us a biased $\mathrm{S} 0 / \pi \sigma^{*} / \pi \pi^{*}$ weighting of $20 / 40 / 40$ 


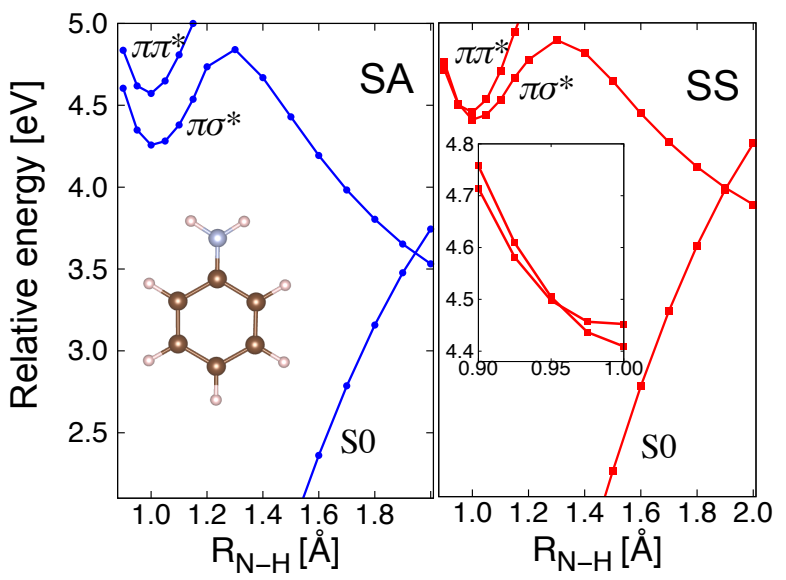

FIG. 2. Potential energy curves showing, for three low-lying singlet states of aniline, the energy of the state after relaxing its geometry under $C_{s}$ symmetry with the $\mathrm{N}-\mathrm{H}$ bond distance $R_{N-H}$ held fixed. SA-CASSCF used a 3 -state SA with equal weights, while both SA-CASSCF and SS-CASSCF employed the $6311++\mathrm{g}(\mathrm{d}, \mathrm{p})$ basis and a $(10 \mathrm{e}, 9 \mathrm{o})$ active space containing seven $\pi / \pi^{*}$ orbitals, an $\mathrm{N}-\mathrm{H} \sigma$ orbital, and an $\mathrm{N}-\mathrm{H} \sigma^{*}$ orbital.



$0.9 \AA$

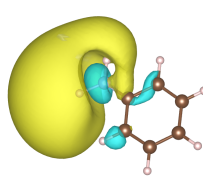

$1.2 \AA$

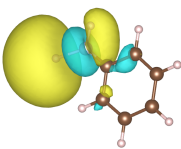

$1.5 \AA$

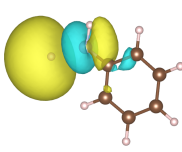

$1.7 \AA$
FIG. 3. The $\sigma^{*}$ orbital of aniline in the $\mathrm{SS}-\mathrm{CASSCF}{ }^{1} \pi \sigma^{*}$ state at various $\mathrm{N}-\mathrm{H}$ bond distances.

and re-optimize the two excited state geometries with $R_{N-H}$ fixed at $0.95 \AA$, the excited state energies do not get closer together. This finding strongly suggests that dynamic weighting would not help here, which is not surprising as, again, the issue is that the two states have significantly different dipoles and so require different postexcitation orbital relaxations. By instead going in for fully state-specific orbitals, which can be seen as the logical endpoint of biased weighting, the different states get the orbital relaxations that are appropriate to them, and a crossing is successfully predicted.

Finally, we consider intramolecular charge transfer (ICT) in 4-aminobenzonitrile (ABN), where Segado and co-workers have shown ${ }^{9}$ that SA-CASSCF predicts a qualitatively incorrect structure for the minimum-energy geometry of the ICT state. In addition to a normal fluorescence band associated with a locally-excited (LE) state, UV excitation of this molecule also produces an anomalous fluorescence band due to emission from the ICT state. ${ }^{17,18}$ As shown in Figure 4, there are multiple local minima on the ICT surface that could in principle be relevant. In the planar (PICT) geometry, the amino group, the benzene ring, and the cyano group all lie in the same plane. ${ }^{39}$ In the rehybridized (RICT) geometry,

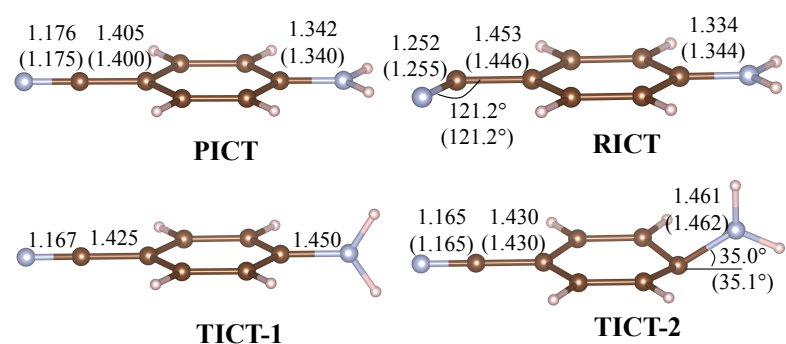

FIG. 4. SS-CASSCF local minima on the ICT surface of ABN, with corresponding SA-CASSCF values from Segado and co-workers ${ }^{9}$ given in parentheses. Both approaches used the cc-pVDZ basis set and a $(12 \mathrm{e}, 11 \mathrm{o})$ active space that contains the benzene $\pi$ and $\pi^{*}$ orbitals, the amino nitrogen lone pair, and the four $\pi$ and $\pi^{*}$ orbitals of the cyano group.

TABLE I. Energy differences $\Delta E$ relative to the S0 minimum (in $\mathrm{kcal} / \mathrm{mol}$ ) and dipoles (in Debye) for ABN's ICT state at different minima on its PES for both SS-CASSCF and the 3 -state SA approach of Segado and coworkers. ${ }^{9}$

\begin{tabular}{cccccc}
\hline \hline \multirow{2}{*}{ States } & \multicolumn{2}{c}{ SS-CASSCF } & & \multicolumn{2}{c}{ SA(3)-CASSCF } \\
\cline { 2 - 3 } \cline { 5 - 6 } & $\Delta E$ & $\mu$ & & $\Delta E$ & $\mu$ \\
\hline S0 & 0 & 6.08 & & 0 & 5.40 \\
PICT & 139.19 & 11.92 & & 146.15 & 11.23 \\
RICT & 140.50 & 13.95 & & 152.77 & 10.45 \\
TICT-1 & 131.70 & 13.66 & & - & - \\
TICT-2 & 128.02 & 10.66 & & 160.93 & 10.76 \\
\hline \hline
\end{tabular}

the cyano group is wagged in the plane of the ring due to a rehybridization of the cyano carbon atom from $s p$ to $s p^{2} \cdot{ }^{40,41}$ In the twisted (TICT) geometries, the amino group is rotated so that its plane is (almost) perpendicular to that of the ring, ${ }^{17,42}$ and may (TICT-1) or may not (TICT-2) involve the group's bond to the ring lying in the ring's plane. ${ }^{9}$ While experiment and high-cost postCASSCF methods agree that the most stable geometry is twisted, ${ }^{17,18}$ Segado and co-workers have shown that SACASSCF instead predicts the fully planar geometry to be more stable by almost $15 \mathrm{kcal} / \mathrm{mol} .{ }^{9}$ As in aniline, this system involves states for which the appropriate postexcitation orbital relaxations differ significantly, and so it is again worth asking whether qualitatively correct predictions really do require expensive post-CASSCF methods or simply that each state be able to enjoy molecular orbitals that have been optimized to suit its needs.

Although Figure 4 reveals that the structures predicted by SS-CASSCF agree with those from SA-CASSCF, Table I shows that the energetics are quite different and that, as anticipated, state-specific orbital relaxation leads to predictions that agree qualitatively with experiment and post-CASSCF methods. In particular, SS-CASSCF agrees with XMS-CASPT2 in predicting that TICT-2 is the most stable ICT structure. ${ }^{9}$ By allowing orbitals to 


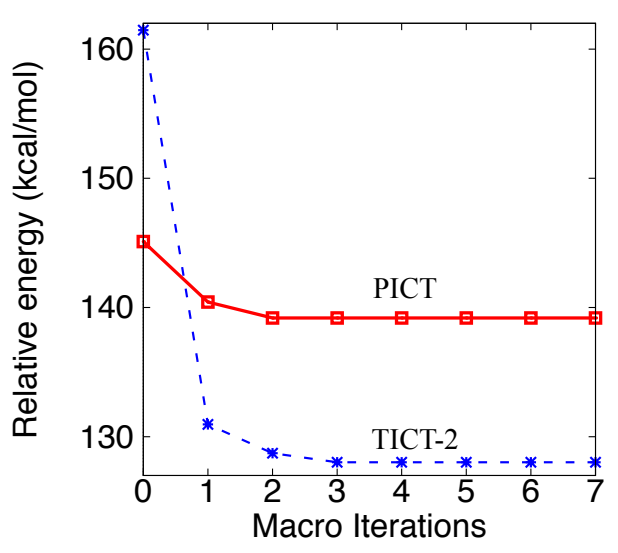

FIG. 5. As an example of how much the SA bias against ABN's ICT state varies with geometry, we plot the lowering of this state's energy during SS-CASSCF optimizations that start from the SA-CASSCF wave functions at both the SS-CASSCF planar (PICT) and twisted (TICT-2) geometry. Energies are reported relative to the $S 0$ minimum.

fully relax following the charge transfer, SS-CASSCF stabilizes the ICT state at all geometries, but the effect is much stronger in the twisted geometries, as shown for example in Figure 5. These results imply that the SA compromise for orbital shapes, which is always worrisome when the non-charge-transfer states (S0, LE) outnumber charge transfer states (ICT), creates a significantly stronger bias against the ICT state at some geometries than at others, ruining the balance that SA is in principle supposed to provide. In contrast, our root-tracking method's ability to tailor orbitals for individual states without losing track of those states during the wave function optimization (see Figure 6) allows for the energetically significant re-polarizations that all orbitals are expected to undergo following a charge transfer. Happily, capturing these effects is sufficient to bring the prediction in line with experiment, again suggesting that one can go a long way in repairing the failures of SA-CASSCF without resorting to the expense of post-CASSCF methods. When one considers that this should be especially true in charge transfer systems and that, in order to separate charge over a significant distance for technological purposes, these systems often contain dozens or even hundreds of atoms, the advantages of improving accuracy without increasing cost become even more desirable.

Conclusion. In summary, we have shown that by providing each excited state with orbitals that are optimal for its needs, qualitative failures in potential energy surfaces can be corrected without significantly increasing computational cost. Moreover, state-specific orbitals dramatically simplify the evaluation of analytic gradients by eliminating the wave function response calculations that are required by state averaged approaches. In particular, we have shown that this state-specific approach, in which collapse to other states is avoided via novel root tracking, makes qualitative improvements over state av-

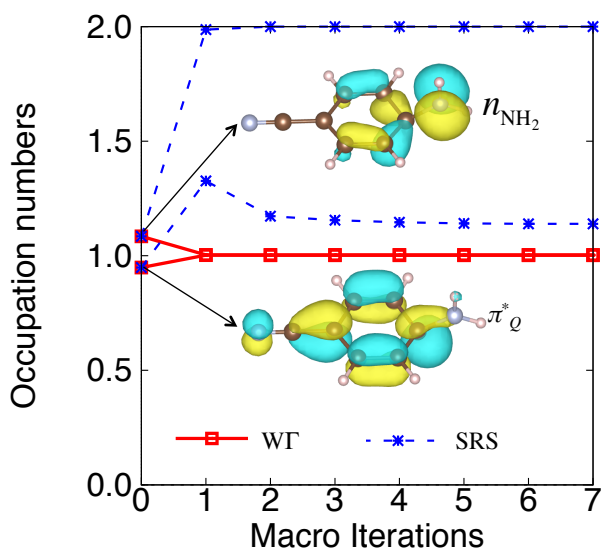

FIG. 6. Orbital occupation changes during SS-CASSCF's wave function optimization of ABN's ICT state at the TICT-2 geometry, with SA-CASSCF used as the guess. Our $W \Gamma$ approach maintains the correct state character, ${ }^{9}$ whereas simple root selection (SRS), which selects the CASCI root based on energy ordering, collapses to a non-charge-transfer state.

eraging in a $\mathrm{C}-\mathrm{S}$ double bond dissociation, in an amino $\mathrm{N}-\mathrm{H}$ dissociation, and in an intramolecular charge transfer geometry relaxation. Our approach's general nature - it requires the same basic ingredients as can be found in ground state CASSCF implementations - should allow these advantages to be enjoyed across a wide variety of excited state applications, with particular promise for cases like charge transfer and core excitation that involve substantial post-excitation orbital relaxation.

Looking forward, there are a number of clear ways in which the methodology can be enhanced to further widen its utility. Thanks to its two-step optimization formulation, it should be quite straightforward to tackle large active spaces with selective CI or the density matrix renormalization group. Combining these approaches with excited-state-specific orbital relaxations looks especially promising for metal-ligand charge transfer complexes, where double-d-shell effects and large ligand $\pi$ systems quite rapidly push the desired active space beyond the reach of conventional solvers. A second obvious priority is enabling inter-state properties like transition dipoles and derivative couplings. Although our approach leads naturally to a situation in which different states are expressed in different molecular orbital bases, making inter-state matrix elements less straightforward, nonorthogonal configuration interaction techniques can be adapted to meet this challenge. These same techniques are also relevant for re-diagonalizing a pair of states near a conical intersection, where the lack of strict orthogonality within our state-specific approach becomes a real concern. With these various improvements, it should be possible to bring the benefits of state-specific orbital optimization, and the qualitative improvements in accuracy that it offers, to the wide array of property predictions on which spectroscopists depend.

Acknowledgement. This work was supported by the 
Early Career Research Program of the Office of Science, Office of Basic Energy Sciences, the U.S. Department of
Energy, grant No. DE-SC0017869. Calculations ran on the Berkeley Research Computing Savio cluster.
* lantrann@berkeley.edu

$\dagger$ eneuscamman@berkeley.edu

1 C. de Graaf and C. Sousa, Chemistry-A European Journal 16, 4550 (2010).

2 C. De Graaf and C. Sousa, International Journal of Quantum Chemistry 111, 3385 (2011).

3 W. Zhang, K. S. Kjær, R. Alonso-Mori, U. Bergmann, M. Chollet, L. A. Fredin, R. G. Hadt, R. W. Hartsock, T. Harlang, T. Kroll, et al., Chemical science 8, 515 (2017).

4 O. S. Wenger, Chemistry-A European Journal 25, 6043 (2019).

5 J. Wu, M. Alías, and C. de Graaf, Inorganics 8, 16 (2020).

${ }^{6}$ L. González, D. Escudero, and L. Serrano-Andrés, ChemPhysChem 13, 28 (2012).

7 F. Aquilante, T. B. Pedersen, V. Veryazov, and R. Lindh, Wiley Interdisciplinary Reviews: Computational Molecular Science 3, 143 (2013).

8 L. Serrano-Andrés and M. Merchán, Journal of Molecular Structure: THEOCHEM 729, 99 (2005).

9 M. Segado, I. Gómez, and M. Reguero, Physical Chemistry Chemical Physics 18, 6861 (2016).

10 W. J. Glover, The Journal of Chemical Physics 141, 171102 (2014).

11 M. P. Deskevich, D. J. Nesbitt, and H.-J. Werner, The Journal of chemical physics 120, 7281 (2004).

12 W. Glover, A. Paz, W. Thongyod, and C. Punwong, The Journal of chemical physics 151, 201101 (2019).

13 L. N. Tran, J. A. Shea, and E. Neuscamman, Journal of chemical theory and computation 15, 4790 (2019).

14 D. Hollas, L. Sistik, E. G. Hohenstein, T. J. Martínez, and P. Slavicek, Journal of chemical theory and computation 14, 339 (2018).

15 P. Slavíček and T. J. Martínez, The Journal of chemical physics 132, 234102 (2010).

16 J. Yang, X. Zhu, T. J. Wolf, Z. Li, J. P. F. Nunes, R. Coffee, J. P. Cryan, M. Gühr, K. Hegazy, T. F. Heinz, et al., Science 361, 64 (2018).

17 Z. R. Grabowski, K. Rotkiewicz, and W. Rettig, Chemical reviews 103, 3899 (2003).

18 R. Misra and S. P. Bhattacharyya, Intramolecular Charge Transfer: Theory and Applications (John Wiley \& Sons, 2018).

19 M. Sala, O. M. Kirkby, S. Guérin, and H. H. Fielding, Physical Chemistry Chemical Physics 16, 3122 (2014).

20 J. Ray and S. G. Ramesh, Chemical Physics 515, 77 (2018).

21 Y. Yamaguchi, A new dimension to quantum chemistry: analytic derivative methods in ab initio molecular electronic structure theory (Oxford University Press, USA, 1994).

22 A. A. Granovsky, The Journal of Chemical Physics 143,
231101 (2015).

23 T. J. Dudley, R. M. Olson, M. W. Schmidt, and M. S. Gordon, Journal of computational chemistry 27, 352 (2006).

24 A. Domingo, M. À. Carvajal, C. de Graaf, K. Sivalingam, F. Neese, and C. Angeli, Theoretical Chemistry Accounts 131, 1264 (2012).

25 S. D. Pineda Flores and E. Neuscamman, J. Phys. Chem. A 123, 1487 (2018).

${ }^{26}$ Q. Sun, T. C. Berkelbach, N. S. Blunt, G. H. Booth, S. Guo, Z. Li, J. Liu, J. D. McClain, E. R. Sayfutyarova, S. Sharma, et al., WIREs Comput. Mol. Sci. 8, e1340 (2018).

27 L.-P. Wang and C. Song, The Journal of chemical physics 144, 214108 (2016).

${ }^{28}$ https://github.com/jhrmnn/pyberny.

29 H.-J. Werner, P. J. Knowles, G. Knizia, F. R. Manby, and M. Schütz, Wiley Interdisciplinary Reviews: Computational Molecular Science 2, 242 (2012).

30 Z. Xu, N. Luo, S. Federman, W. M. Jackson, C.-Y. Ng, L.P. Wang, and K. N. Crabtree, The Astrophysical Journal 882, 86 (2019).

31 R.-Z. Mao, F. Guo, D.-C. Xiong, Q. Li, J. Duan, and X.-S. Ye, Organic letters 17, 5606 (2015).

32 T. Ganguly and A. Majumdar, Inorganic Chemistry (2020).

33 M. N. Ashfold, M. Bain, C. S. Hansen, R. A. Ingle, T. N. Karsili, B. Marchetti, and D. Murdock, The journal of physical chemistry letters 8, 3440 (2017).

34 G. M. Roberts and V. G. Stavros, Chemical Science 5, 1698 (2014).

35 G. A. King, T. A. Oliver, and M. N. Ashfold, The Journal of chemical physics 132, 214307 (2010).

${ }^{36}$ G. M. Roberts, C. A. Williams, J. D. Young, S. Ullrich, M. J. Paterson, and V. G. Stavros, Journal of the American Chemical Society 134, 12578 (2012).

37 F. Wang, S. Neville, R. Wang, and G. Worth, The Journal of Physical Chemistry A 117, 7298 (2013).

38 W. R. Jhang, H. Y. Lai, Y.-C. Lin, C. Lee, S.-H. Lee, Y.-Y. Lee, C.-K. Ni, and C.-M. Tseng, The Journal of Chemical Physics 151, 141101 (2019).

39 S. I. Druzhinin, N. P. Ernsting, S. A. Kovalenko, L. P. Lustres, T. A. Senyushkina, and K. A. Zachariasse, The Journal of Physical Chemistry A 110, 2955 (2006).

40 A. L. Sobolewski and W. Domcke, Chemical physics letters 259, 119 (1996).

41 A. L. Sobolewski and W. Domcke, Chemical physics letters 250, 428 (1996).

42 K. Rotkiewicz, K. Grellmann, and Z. Grabowski, Chemical Physics Letters 19, 315 (1973). 